\title{
Three-Dimensional Optical Transformer - Highly Efficient Nanofocusing Device
}

\author{
Hyuck Choo ${ }^{1,2}$, Matteo Stafarroni ${ }^{2}$, Tae Joon Seok ${ }^{2}$, Jeffrey Bokor ${ }^{1,2}$, Ming Wu ${ }^{2}$, P. J. Schuck ${ }^{1}$, S. Cabrini ${ }^{1}$, \\ and Eli Yablonovitch ${ }^{2}$ \\ 1. Molecular Foundry, Lawrence Berkeley National Laboratory, Berkeley California \\ 2. Department of Electrical Engineering and Computer Sciences, University of California Berkeley \\ Email:hchoo@lbl.gov
}

\begin{abstract}
Using electron-beam-induced deposition and focused-ion-beam milling, we have fabricated and demonstrated a nanofocusing optical transformer with a 3-dimensionally tapered tip. At the tip, the light is confined to $13-$ by-80-nm area with intensity enhancement exceeding 1500 . (C)2010 Optical Society of America

OCIS codes: 350.4238 Nanophotonics and Photoni Crystals; 240.6680 Surface Plasmons
\end{abstract}

\section{Introduction}

Scientific and commercial applications of optical nanofcousing devices have attracted a lot of research efforts in recent years. Sub-diffraction-limited microscopy, nano-photolithography, tip-based nanofabrication, and heatassisted magnetic recording are a few examples. To realize these applications, nanofocusing devices must be able to confine light into sub-100-nm space with great efficiency. Previously, researchers have achieved nanofocusing using scanning-tunneling-microscope (STM) tips, tapered plasmonic pins, enhanced transmission apertures, and tapered fiber probes [1-4]. Most of these techniques achieve $\leq 100 \mathrm{~nm}$ in spot diameter. However, their low efficiency makes them unsuitable for applications that require nanoscale spot sizes as well as high optical-energy throughput, such as heat-assisted magnetic recording (HAMR) for achieving data density beyond $1 \mathrm{Tbits} / \mathrm{in}^{2}$.

\section{Optical Transformer Concept, Fabrication, and Characterizations}

Using electron-beam-induced deposition and focused ion-beam milling [5], we have fabricated plasmonic optical transformers with a tapered tip (Fig. 1). The fabricated transformers are composed of $\mathrm{Au} / \mathrm{SiO}_{2} / \mathrm{Au}$ layers and have tips that linearly taper in two dimensions. The thickness of the $\mathrm{SiO}_{2}$ layer, fabricated using e-beam induced deposition, decreases from $200 \mathrm{~nm}$ down to $<15 \mathrm{~nm}$, which is an important feature of the transformer.

The concept of the optical transformer and its optimized dimensions (from COMSOL simulation) are shown in Fig. 2 (a) and (b). The optical transformer is an impedance transformer at optical frequency. The gradually decreasing vertical gap and horizontal width of the structure along the optical axis increase the kinetic impedance and makes it possible to focus lights into a few nanometer dimensions with a very low loss. The two major advantages of the optical transformer over conventional plasmonic antennas are (a) much improved enhancement of electric field $\left(>10^{3}\right.$ with an efficient optical coupler); and (b) excellent impedance match to analyte molecules. Due to the intrinsic properties of metal-insulator-metal waveguides, the optical focus size is determined by the dimensions of the dielectric layer, making it possible to achieve sub-10-nm spot sizes (Fig. 2 (c)).

Our fabrication process is illustrated in Fig. 3. We first deposit a 50-nm-thick gold layer (Fig. 3 (a)) on a substrate. The dielectric material, which is $\mathrm{SiO}_{2}$, is deposited using electron-beam induced deposition (Fig. 3 (b)). The tapering geometry is achieved by controlling the scan (deposition) area and the dose of the electron beam and by performing multi-layer depositions. The process has enabled us to obtain tapering angles between 5 - 45 degrees and deposit layers as thin as $5 \mathrm{~nm}$. Following the $\mathrm{SiO}_{2}$ deposition, another 50-nm-thick gold layer is deposited (Fig. $3(\mathrm{c})$ ). The final shape of the transformer is defined by performing focused ion-beam milling (Fig. $3(\mathrm{~d})$ ).

Using 120-fs Ti-sapphire-laser pulses at $830 \mathrm{~nm}$ (focused down to a diffraction limited spot, FWHM $\sim 400 \mathrm{~nm}$ ), we excited at the base of the transformer and collected the two-photon luminescence from the base (Fig. 4 (a) and from the tapered tip (Fig. 4 (b)). As a reference, we also measured the two-photon luminescence from a gold surface (with peak-to-peak roughness of $8 \mathrm{~nm}$ ) using approximately twenty times higher laser power (Fig. 4 (c)).

To calculate the intensity enhancement factor using the equation (1) from the reference 6, we compared the TPPLemission intensity measured at the tip of the optical transformer to that from the flat gold film (peak-to-peak roughness of $8 \mathrm{~nm}$ ). We used the COMSOL simulation to calculate the active mode volume inside the tip as well as the light-coupling efficiency $(8.6 \%)$ into the transformer. For the gold film, we assumed the excitation-spot diameter of $400 \mathrm{~nm}$ and skin depth of $25 \mathrm{~nm}$ to calculate the active mode volume. The calculated intensity enhancement is 1502 . To our knowledge, these are the highest enhancement values that have been reported for 13by-80-nm spot size. 


\section{CFI5.pdf}

\section{References}

[1] L. Novotny, et al., “Theory of Nanometric Optical Tweezers,” Physical Review Letters, 79, 645 - 648 (1997)

[2] R. Ruppin, "Effect of non-locality on nanofocusing of surface plasmon field intensity in a conical tip," Physics Letters A, 340, 299-302 (2005)

[3] W. L. Barnes, et al., "Surface plasmon subwavelength optics," Nature, 424, 824-830 (2003)

[4] T. Yatsui, et al., "Increasing throughput of a near-field optical fiber probe over 1000 times by the use of a triple-tapered structure," APL, 73, 2090- 2092 (1998)

[5] I. Utke, et al., "Gas-assisted focused electron beam and ion beam processing and fabrication," JVST B, 26, 1197-1276 (2008)

[6] P. J. Shuck, et al., "Improving the Mismatch between Light and Nanoscale Objects with Gold Bowtie Nanoantennas," Physical Review Letters, 94, 017402-1-4 (2005)
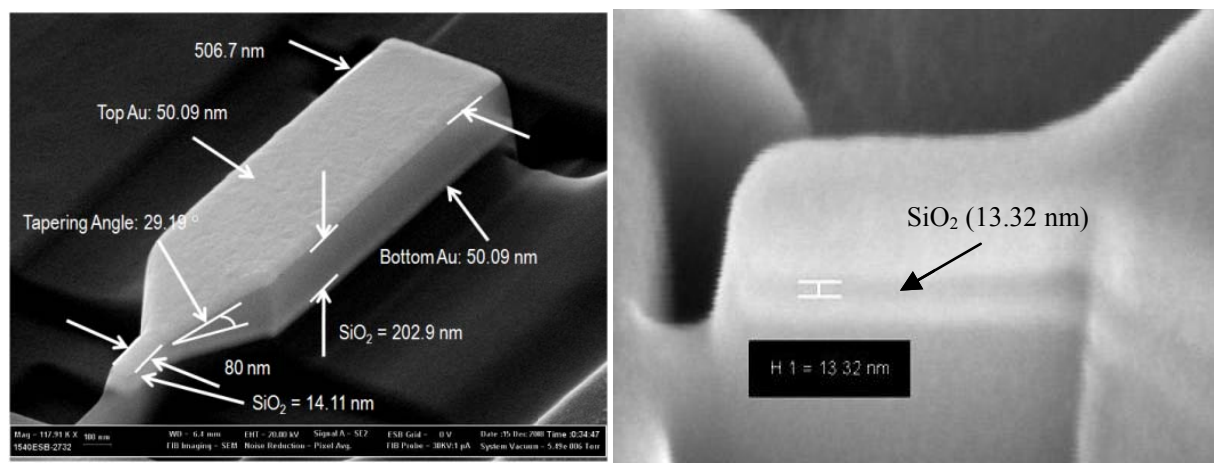

Fig. 1. SEM images: fabricated optical transformer and side view of the tapered tip
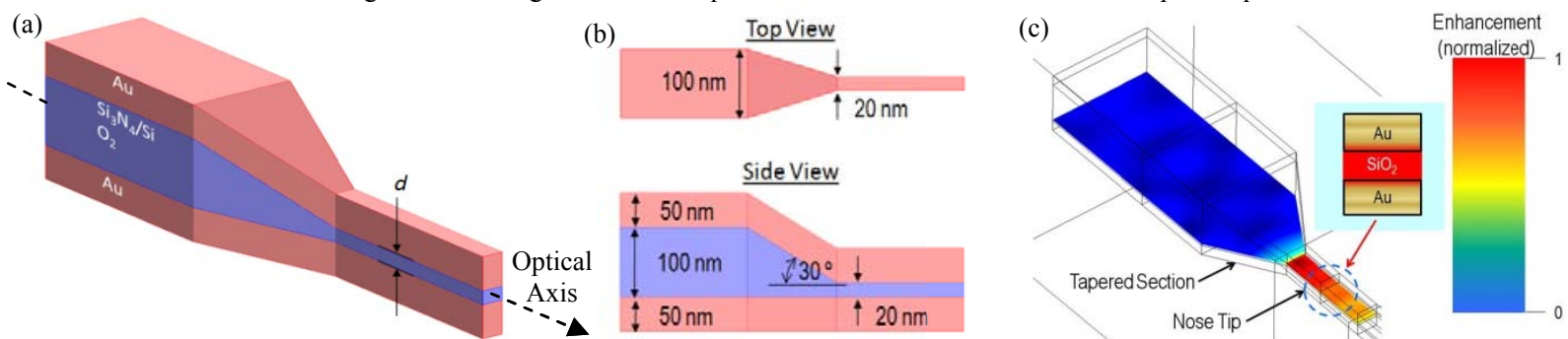

Fig. 2. (a) The concept of an optical transformer; (b) optimized dimensions (from COMSOL simulation) - A smaller plate separation at the tapered end will result in stronger e-field enhancement; and (c) final focus dimension (from COMSOL simulation)

(a)

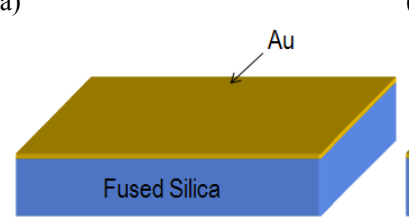

(b)

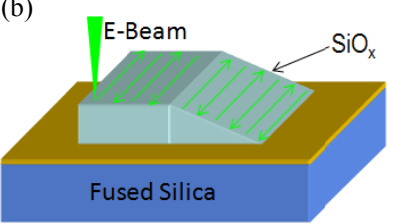

(c)

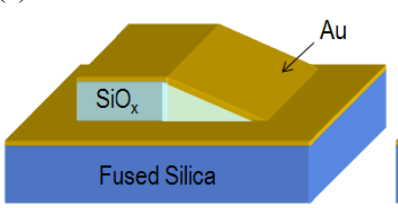

(d)

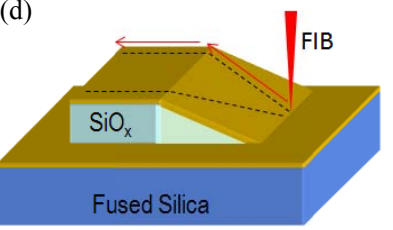

Fig. 3. Fabrication process: (a) Au deposition; (b) electron-beam induced deposition of $\mathrm{SiO}_{2}$; (c) Au deposition; and (d) final patterning using focused ion beam

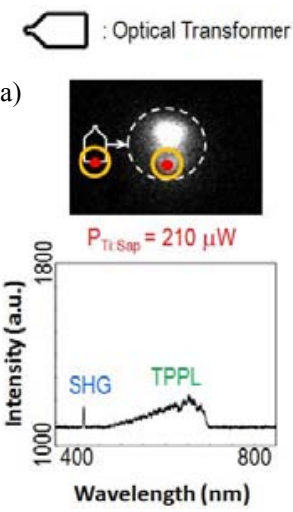

: Laser-excitation location

: Collecting Pinhole location

(b)
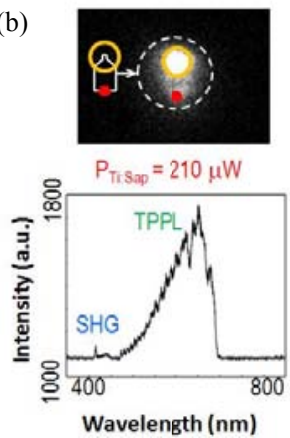

(c)
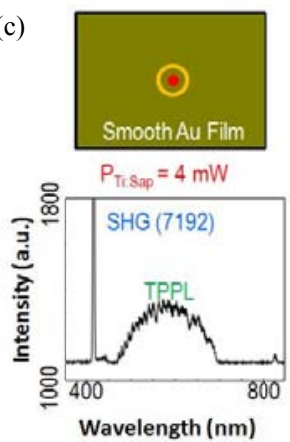

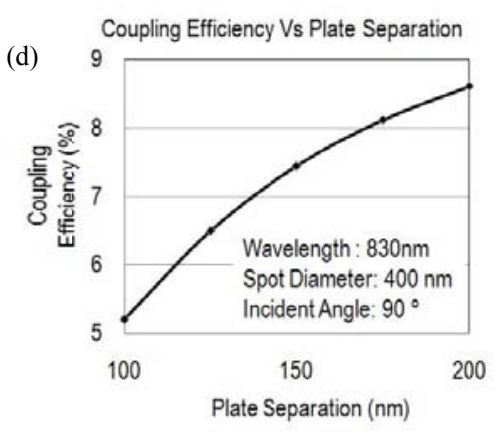

Fig. 4. TPPL measurements: (a) Excitation and collection at the base; (b) excitation at the base and collection at the tip; (c) TPPL measured on a smooth gold film (peak-to-peak roughness of $8 \mathrm{~nm}$ ); and (d) light-coupling efficiency into the optical transformer at the base at normal incidence 